\title{
The effect of a clinic-based behavioral intervention in promoting enrolment in a text-message tobacco cessation program at a rural primary health clinic in Delhi, India
}

\author{
Saurav Basu', Priyanka Yadav', Bratati Banerjee', Ankit Yadav'
}

\begin{abstract}
INTRODUCTION The mCessation is a text-message service for quitting tobacco in India, associated with high quit rates but low rates of enrolment. We determined if a brief behavioral intervention package (BBPMC) can promote the enrolment of male tobacco users in the mCessation program (MCP)

METHODS We conducted a quasi-experimental study (single-arm) at a rural primary health clinic in Delhi, India, January-April 2019. We enrolled adult male current tobacco users after screening for eligibility. A trained medical physician administered the BBPMC explaining to patients the major health risks causally associated with tobacco use, along with information on the MCP and the way to register for the service.

RESULTS We recruited a total of 159 tobacco users, mean age 44.5 years $(\mathrm{SD}=14.3)$ with median tobacco use duration of 15 years. After administration of the BBPMC, $50(31.2 \%)$ participants registered with the MCP program on-site. On an adjusted analysis, tobacco users with a preexisting intention to quit were three times more likely to enroll in the MCP.

CONCLUSIONS Physicians and other healthcare providers in Indian outpatient health settings should utilize BBPMC like interventions that are easily scalable, require no specialized training, and and allow them to fulfill their obligation to provide a readily accessible tobacco cessation service to their patients.
\end{abstract}



\section{AFFILIATION \\ 1 Department of Community Medicine, Maulana Azad Medical} College, New Delhi, India

CORRESPONDENCE TO

Saurav Basu. Department of Community Medicine, Maulana Azad Medical College, New Delhi, 110002, India. E-mail: saurav.basu1983@gmail.com

\section{KEYWORDS}

smoking cessation, tobacco cessation, behavioral intervention, mCessation

Received: 7 February 2020 Revised: 16 March 2020 Accepted: 8 April 2020

\section{INTRODUCTION}

Tobacco use is responsible for one in ten deaths worldwide with low- and middle-income countries estimated to account for $80 \%$ of the burden by $2030^{1}$. In India, a million deaths annually are attributed to tobacco ${ }^{2}$. The prevalence of tobacco smoking in India is estimated to be $19 \%$ in men, and $2 \%$ in women, while smokeless tobacco use in men is $29.6 \%$ and among women it is $12.6 \%{ }^{3}$. It is well established that smoking cessation is highly beneficial for tobacco smokers and significantly increases their life expectancy ${ }^{4}$. However, smoking trends in India indicate only a modest decline, although more than one in two smokers (55.2\%) plan to quit smoking ${ }^{3,5}$. Nevertheless, even conventional smoking cessation intervention studies conducted in India have reported high quit and harm reduction rates, suggesting a large unmet need for smoking cessation services in India ${ }^{6}$.

Developing countries like India often lack adequate and dedicated modern tobacco cessation clinics, especially in suburban and rural areas ${ }^{7}$. Furthermore, the second round of the global adult tobacco survey reveals that less than half of current smokers $(48.2 \%)$ had been advised to quit smoking by a healthcare provider $(\mathrm{HCP})^{3}$. Assisting and arranging tobacco cessation services for their patients can also be problematic for HCPs working in resource-constrained settings due to factors involving patient load, lack of training, and short duration of the consultation. Self-efficacy in 
imparting useful and effective health information by doctors to their patients can also be suboptimal ${ }^{8}$.

There is growing recognition that tailored mobile phone-based text-messages assisting with quitting tobacco are highly cost-effective public health interventions that can significantly enhance the accessibility, affordability, and availability of cessation services ${ }^{9,10}$. India, in partnership with the World Health Organization and the International Telecommunication Union, launched a short text message (SMS) based mobile health mCessation program (MCP) in $2015^{11}$. The MCP is available free of cost to Indian residents in the Hindi and English languages. Beneficiaries can be registered to the service by sending a text message to a toll-free telephone number, and no internet access is required. The MCP tailors a tobacco cessation package by querying the user's tobacco use habits and their expected, planned timeline for quitting. More than a million users have availed themselves of the MCP with $19 \%$ successful quit rates observed at $\geq 30$ days and 4-6 months after completion of the program ${ }^{12}$.

We assume that awareness and acceptability of the MCP can be further enhanced in conjunction with HCPs who can explain and recommend the service to their patients who are current tobacco users. We, therefore, conducted this study to generate evidence regarding the feasibility of integrating the MCP in routine outpatient care in a primary health setting. Furthermore, we determined if a clinic-based, brief behavioral intervention package can promote the enrolment of tobacco-using patients in the MCP.

\section{METHODS}

\section{Design and setting}

We conducted a quasi-experimental (single arm-trial) study for four months from January to April 2019. We recruited participants from the outpatient setting of a rural primary health clinic in Delhi, India, catering to a population of approximately 5500 residents, and affiliated to a prominent medical college in Delhi, India. The clinic receives, on average, 70 patients per day and is staffed by a medical officer, four to six resident doctors, including those in training in the speciality of Community Medicine, and a public health nurse.

\section{Study participants}

Patients visiting the clinic for any health complaint were queried regarding their current tobacco usage status by the treating physician. Patients that were tobacco users were subsequently directed to one of the investigators at the clinic to assess eligibility for enrolment.

Inclusion criteria were: adult male patients reporting for any medical complaint at the outpatient department (OPD) and used tobacco products in any form. Furthermore, we considered an operational definition of current tobacco smokers as those who reported having consumed at least 100 cigarettes or bidis (hand-rolled cigarettes) during their lifetime and were current smokers. Current smokeless tobacco users were defined as those who reported consuming any such products in the previous three days. Exclusion criteria were: males who had previously registered with the MCP, those not having mobile phones, or patients with any significant physical (visual impairment, severe arthritis) or mental disability (dementia) that was likely to hamper their ability to use their mobile phones to avail themselves of the MCP.

Patients who were unable to read SMS text messages but were willing to register with the MCP with the assistance of any caregivers in the family were permitted to enroll in the study. Women patients were not screened for participation eligibility since their tobacco use prevalence is known to be very low, as tobacco use is considered socially stigmatizing in the particular study setting.

\section{Standard care}

All physicians in India are expected to counsel their patients to quit tobacco, but there exist no specific guidelines and recommendations on how to arrange help for the patient when required locally. Similarly, at the study site, the treating physician may query the patient regarding their tobacco use practices and instruct them to quit. However, no information regarding the MCP was being provided as part of usual care.

\section{Intervention}

A brief behavioral-change package for promoting enrolment in the MCP program (BBPMC) was developed specifically for the study. The BBPMC was administered to the participants by a designated medical physician (one male and one female) directly, and it was of two minutes duration. The physician 
briefly explained the addiction potential and harmful consequences of tobacco use to the participants. The participants were informed of the risks of lung and oral cancer, and the increased risk of heart disease and stroke among tobacco users. They were further told of the benefits of tobacco cessation, in terms of the significant reduction in the risks of tobacco-related diseases with benefits accruing within one year of quitting and translating into a gain in the years of life expectancy with each cumulative year since cessation. Finally, the participants were explained the purpose of the MCP along with its advantages and the ways to register and utilize it for quitting tobacco.

\section{Outcomes}

The primary outcome of the study was the proportion of tobacco users who enrolled in the MCP program after the administration of the BBPMC. Secondary outcomes were prior awareness of the participants regarding the MCP program and their readiness to enroll in the MCP program.

\section{Outcome measurement}

The primary outcome was ascertained on the spot, based on the participant's immediate response in terms of either successfully enrolling in the MCP program on-site, keeping in abeyance the decision to enroll, or refusing to enroll. Secondary outcomes were assessed through specific questions asked during faceto-face interviews using a patient interview schedule.

\section{Sample size and sampling}

According to data from the second round of the Global Adult Tobacco Survey (2016-17) in India, $3 \%$ of tobacco users utilized the $\mathrm{MCP}^{3}$. We pretested the BBPMC in 10 male tobacco users at a different site and observed $30 \%$ enrolment in the MCP on the application of the intervention. Assuming 10\% of tobacco users would enroll in the MCP in the absence of any intervention, and that $30 \%$ tobacco users would enroll after receiving the BBPMC, the sample size was estimated to be 158 , at $90 \%$ power and $95 \%$ confidence level, as per a binary outcome superiority trial formula ${ }^{13}$. Participants were enrolled in the trial via consecutive sampling.

\section{Study instrument}

A pretested patient interview schedule in the local language, Hindi, was used for data collection through face-to-face interviews. The participants were asked questions regarding: 1) sociodemographic characteristics (age, educational level, socioeconomic status); 2) existing tobacco consumption (both smoking and smokeless types); 3 ) awareness of diseases caused by tobacco use (lung cancer and oral cancer); 4) intention to quit tobacco, using a single close-ended question (i.e. 'Have you considered quitting any form of tobacco in the previous six months?' with responses coded dichotomously as 'yes' or 'no'); 5 ) instructions given to quit tobacco by any healthcare provider; 6) self-efficacy towards mobile phone use for text messaging, assessed with a single close-ended question (i.e. 'Do you know how to send SMS messages?'); 7) perceived usefulness of the MCP program, measured on a 4-point rating scale (not useful, neutral, somewhat useful, useful); and 8) perceived barriers in the use of the MCP.

The previously validated BG Prasad scale was applied to determine the socioeconomic status (SES) of the participants for rural populations based on the per capita income, updated for current income criteria $^{14}$.

The male patients visiting the OPD were initially screened for eligibility and were interviewed face-to-face using the interview schedule after obtaining their written and informed consent. All the participants were subsequently administered the BBPMC. The study was exempted from full review by the Institutional Ethics Committee of the medical college, and prospectively registered with the clinical trial registry of India (CTRI/2019/01/016849), dated 2 January 2019.

\section{Statistical analysis}

We analyzed the data using IBM SPSS Version 25. Categorical data were expressed in frequency and proportions, and continuous data as mean and standard deviation. The association between categorical variables was assessed using the $\chi^{2}$ test. A p-value $<0.05$ was considered statistically significant. A binary logistic regression analysis was conducted by including those variables that were significantly associated with enrolment for MCP post-intervention. The final model was tested for goodness-of-fit by the Hosmer-Lemeshow test. 


\section{RESULTS}

\section{Participant characteristics}

We recruited a total of 159 male tobacco users in the study. The mean (SD) age of the participants was 44.5 (14.3) years, ranging from 18 to 80 years. A total of $62(39 \%)$ participants were educated at-least until high school. A majority (75.5\%) of the participants reported having visited a health facility within the last 12 months. None of the participants was previously diagnosed with lung cancer. Ownership of a personal mobile phone was reported by $129(81.1 \%)$ participants.

\section{Tobacco usage in the participants}

The mean (SD) duration of tobacco usage in the participants was 18.1 (13.9) years, while the median duration was 15 years. All the participants reported being everyday tobacco users. The mode of consumption of tobacco was reported as only cigarettes by $15(9.4 \%)$, only bidis by $69(43.4 \%)$, both smoking and smokeless forms by $17(10.7 \%)$ and only smokeless forms by 58 (36.5\%). The mean (SD) number of cigarettes/bidis consumed per day by the participants was 8.8 (7.7). Participant awareness of a causal relation between tobacco smoking and lung cancer was present in 116 (73\%), smokeless tobacco use and oral cancer in 85 (53.5\%), and both in 81 (50.9\%) participants.

\section{Effectiveness of the intervention (BBPMC)}

Only $10(6.3 \%)$ participants were aware of the
MCP program before the intervention. After the administration of the BBPMC, 50 (31.2\%) participants registered with the MCP program on-site. Among those who did not register with the service, 57 (35.8\%) of the participants reported they would consider enrolment in the future, $13(8.2 \%)$ believed MCP would not be useful in helping them quit tobacco, 14 (8.8\%) did not want to quit tobacco, and 25 (15.7\%) were not confident in reading text messages on their mobile phones. However, $22.2 \%$ of participants who registered for the MCP, despite lacking self-efficacy in reading and understanding mobile text-messages, believed they could utilize familial caregiver support to benefit from the service. The perceived usefulness of MCP was rated by the participants as: being useful (23.3\%), somewhat useful (28.3\%), neutral (20.1\%), and not useful $(28.3 \%)$.

Post-intervention (BBPMC), on bivariate analysis, showed that higher educational status ( $\geq 10$ years), duration of tobacco use $\leq 15$ years, prior contemplation for quitting smoking in the previous six months, and confidence in reading text messages on the mobile phone, were found to be statistically significant predictors $(p<0.05)$ of participant enrolment in the MCP program (Table $1)$. On binary logistic regression analysis, the model was statistically significant $(\mathrm{p}<0.001)$ and correctly classified $68.6 \%$ of the cases. Among the four predictor variables, only the presence of prior contemplation for quitting tobacco was statistically significant $(\mathrm{p}=0.008)$ (Table 2$)$.

Table 1. Predictors of the effectiveness of a brief behavior change intervention package (BBPMC) on mCessation program enrolment in male tobacco users in Delhi, India $(\mathrm{N}=159)$

\begin{tabular}{|c|c|c|c|c|}
\hline \multirow[t]{2}{*}{ Characteristics } & \multicolumn{4}{|c|}{ mCessation enrolment status } \\
\hline & Total & $\begin{array}{c}\text { Enrolled } \\
(\mathbf{N}-50) \\
n(\%)\end{array}$ & $\begin{array}{c}\text { Did not enroll } \\
\text { (N }=109) \\
\text { n ( } \%)\end{array}$ & $p$ \\
\hline \multicolumn{5}{|l|}{ Age (years) } \\
\hline$<50$ & $59(37.1)$ & 17 (28.8) & $42(71.2)$ & 0.602 \\
\hline$\geq 50$ & $100(62.9)$ & 33 (33) & 67 (67) & \\
\hline \multicolumn{5}{|l|}{ Education (years) } \\
\hline$<10$ & $97(61)$ & $21(21.6)$ & $76(78.4)$ & $<0.001$ \\
\hline$\geq 10$ & $62(39)$ & $29(46.8)$ & $33(53.2)$ & \\
\hline \multicolumn{5}{|l|}{ SES } \\
\hline 1-3 (High/middle) & $84(52.8)$ & $32(38.1)$ & $52(61.9)$ & 0.062 \\
\hline 4-5 (Low) & $75(47.2)$ & $18(24)$ & $57(76)$ & \\
\hline
\end{tabular}


Table 1. Continued

\begin{tabular}{|c|c|c|c|c|}
\hline \multirow[t]{2}{*}{ Characteristics } & \multicolumn{4}{|c|}{ mCessation enrolment status } \\
\hline & Total & $\begin{array}{l}\text { Enrolled } \\
(\mathbf{N}=50) \\
n(\%)\end{array}$ & $\begin{array}{l}\text { Did not enroll } \\
\begin{array}{c}(\mathrm{N}=109) \\
\text { In }(\%)\end{array}\end{array}$ & p \\
\hline \multicolumn{5}{|l|}{ Smoking status (packs/day) } \\
\hline$\leq 1$ & $26(16.3)$ & $6(23)$ & $20(77)$ & 0.364 \\
\hline$>1$ & $133(83.5)$ & $44(33)$ & $89(67)$ & \\
\hline \multicolumn{5}{|l|}{ Duration tobacco use (years) } \\
\hline$\leq 15$ & $88(55.3)$ & $34(38.6)$ & $54(61.3)$ & 0.030 \\
\hline$\geq 16$ & $71(44.7)$ & $16(22.5)$ & $55(77.5)$ & \\
\hline \multicolumn{5}{|l|}{ Type of tobacco } \\
\hline Smoke with/without smokeless & $101(63.5)$ & $33(32.7)$ & $68(67.3)$ & 0.660 \\
\hline Smokeless only & $58(36.5)$ & $17(29.3)$ & $41(70.7)$ & \\
\hline \multicolumn{5}{|l|}{ Tobacco related harm awareness } \\
\hline Present & $81(50.9)$ & $24(29.6)$ & $57(70.4)$ & 0.615 \\
\hline Absent & $78(49.1)$ & $26(33.3)$ & $52(66.7)$ & \\
\hline \multicolumn{5}{|c|}{ HCP advised quitting in previous 12 months } \\
\hline Yes & $44(27.7)$ & $16(36.4)$ & $28(63.6)$ & 0.448 \\
\hline No & $115(72.3)$ & $34(29.5)$ & $81(70.4)$ & \\
\hline \multicolumn{5}{|c|}{ Prior quitting contemplated in the previous 6 months } \\
\hline Yes & $89(56)$ & $38(42.7)$ & $51(57.3)$ & 0.001 \\
\hline No & $70(44)$ & $12(17.2)$ & $58(82.8)$ & \\
\hline \multicolumn{5}{|c|}{ Sought help to quit in previous 6 months } \\
\hline Yes & $15(9.4)$ & $8(53.3)$ & $7(46.7)$ & 0.078 \\
\hline No & $144(90.6)$ & $42(29.2)$ & $102(70.8)$ & \\
\hline \multicolumn{5}{|c|}{ Comfortable with reading text messages } \\
\hline Yes & 69 (43.4) & $30(43.5)$ & $39(56.5)$ & 0.006 \\
\hline No & $90(56.6)$ & $20(22.2)$ & 70 (77.8) & \\
\hline
\end{tabular}

SES: socioeconomic status. HCP: healthcare provider.

Table 2. Logistic regression analysis of predictors associated with mCessation program enrolment in male tobacco users after intervention (BBPMC), Delhi, India

$\begin{array}{lccc}\text { Predictor variable } & \text { OR }\left(95^{\circ} \circ \mathrm{CI}\right) & \text { IOR }\left(95^{\circ} \circ \mathrm{CI}\right) & \text { P } \\ \text { Prior quit contemplation present } & 2.49(1.41-4.40) & 2.867(1.31-6.26) & 0.008 \\ \text { Comfortable with reading text-messages } & 1.96(1.22-3.13) & 1.490(0.65-3.41) & 0.283 \\ \text { Lower educational status }<10 \text { years } & 0.46(0.29-0.73) & 0.455(0.20-1.04) & 0.061 \\ \text { Tobacco use duration } \leq 15 \text { years } & 1.7(1.0-2.84) & 1.656(0.78-3.51) & 0.189\end{array}$

OR: odds ratio. AOR: adjusted odds ratio. $\mathrm{Cl}$ : confidence interval.

\section{DISCUSSION}

Achieving tobacco cessation is essential to prevent large-scale morbidity and mortality from tobaccorelated diseases. The evidence from our study indicates that a brief behavioral-change intervention (BBPMC) by a healthcare provider can significantly promote enrolment in the mCessation program (MCP) among rural adult males in India. Furthermore, nearly one in five participants that lacked confidence in reading text messages still enrolled for MCP by relying on familial support to utilize the service for quitting tobacco.

In our study, tobacco users with a preexisting intention to quit were nearly three times more likely to respond positively to the BBPMC by registering with the MCP, compared to smokers lacking prior 
intention to quit. Smokers with more years of education were also more likely to enroll for the MCP, which agrees with previous evidence linking literacy with enhanced intention to quit ${ }^{15}$. The present study found that adequate knowledge of smoking-related illnesses was absent in $27 \%$ of participants, much higher than the $5 \%$ reported by GATS- 2 .

Previous studies have reported that an increase in knowledge about tobacco risks and harmful effects can increase the quit attempt rates in smokers ${ }^{16}$. Furthermore, healthy smokers are often unaware of smoking cessation services, even in developed nations ${ }^{17}$. The BBPMC explained to the participants the known cancer-related health risks due to tobacco use and increased their awareness regarding the MCP, which was very low $(6.3 \%)$ at baseline. These factors probably explain the success of the BBPMC in significantly improving enrolment in the MCP by catering for an unmet need and providing tobacco users with a readily available facility for cessation.

Our study findings have important implications for public health efforts focused on promoting tobacco cessation in India. First, there exists a significant demand for text-messaging services like the MCP that help people in quitting tobacco. Consequently, there is a need to effectively integrate the MCP with India's National Tobacco Control Program (NTCP). Moreover, there is low awareness of MCP among tobacco users in India, which needs to be enhanced through information, education, and communication (IEC) campaigns. Second, healthcare providers should be sensitized and trained to deliver BBPMC like interventions to their patients with tobacco addiction, as part of standard care.

\section{Limitations}

There are certain study limitations. First, the study was conducted at a single clinic site, and the baseline characteristics of the study participants in terms of knowledge about smoking risks and prior physiciandirected quit advice were much lower compared to the GATS-2 estimates ${ }^{3}$. This highlights the lack of representativeness of the sample and limits the generalizability of the study findings. Second, the study participants were not observed prospectively, which precluded the estimation of quit and harm reduction rates. Third, we also could not measure the proportion of tobacco users who may have later registered for the MCP. Fourth, we did not assess factors that were driving the intention to quit, including the prevalence of debilitating lung diseases like chronic obstructive pulmonary disease (COPD) in the study participants. Finally, it could be argued that higher enrolments in the MCP observed in our study were an outcome of assistance received by the patients from their caregivers or attendants, which invariably introduces uncertainty. However, we ensured that the decision to enroll in the MCP was the participant's. Furthermore, the role of family support in successfully applying behavioral interventions is well-established ${ }^{18,19}$. Hence, text-message interventions in settings like that of our study, especially in elderly patients, should be interpreted as more family-centered interventions rather than alone with the individual.

\section{CONCLUSIONS}

Physicians and other healthcare providers in Indian outpatient health settings should utilize BBPMC like interventions that are easily scalable, require no specialized training, and allow healthcare providers to fulfill their obligation to provide a readily accessible tobacco cessation service to their patients. Future studies should evaluate the effectiveness of the BBPMC in promoting enrolment in the MCP and the quit status of tobacco-using patients in different levels of healthcare. The application of the BBPMC should also be considered in remote and resourceconstrained settings that are more likely to be deficient in dedicated smoking cessation clinics and services. The effect of more elaborate counseling strategies as part of a modified BBPMC for enhancing patient acceptability of the MCP should also be evaluated. The utilization of India's vast network of frontline health workers for increasing awareness of the MCP and capacity for delivering BBPMC like interventions to tobacco users in the community may also hold promise. The applicability of the BBPMC in high burden vulnerable groups like tuberculosis patients during initiation and continuation of antitubercular treatment also requires ascertainment ${ }^{20}$. Finally, the cost-effectiveness of integrating BBPMC like interventions in the health system also warrants consideration.

\section{REFERENCES}

1. Reitsma MB, Fullman $\mathrm{N}, \mathrm{Ng} \mathrm{M}$, et al. Smoking prevalence and 
attributable disease burden in 195 countries and territories, 1990-2015;2015: a systematic analysis from the Global Burden of Disease Study 2015. Lancet. 2017;389(10082):18851906. doi:10.1016/S0140-6736(17)30819-X

2. Mishra GA, Pimple SA, Shastri SS. An overview of the tobacco problem in India. Indian J Med Paediatr Oncol. 2012;33(3):139-145. doi:10.4103\%2F0971-5851.103139

3. Tata Institute of Social Sciences (TISS), Mumbai and Ministry of Health and Family Welfare, Government of India. Global Adult Tobacco Survey: India 2016-2017. http://www.indiaenvironmentportal.org.in/files/file/ GATS_.pdf. Accessed March 15, 2020.

4. Gellert C, Schöttker B, Brenner H. Smoking and allcause mortality in older people: systematic review and meta-analysis. Arch Intern Med. 2012;172(11):837-844. doi:10.1001/archinternmed.2012.1397

5. Mishra S, Joseph RA, Gupta PC, et al. Trends in bidi and cigarette smoking in India from 1998 to 2015, by age, gender and education. BMJ Glob Health. 2016;1(1):e000005. doi:10.1136\%2Fbmjgh-2015-000005

6. Kumar MS, Sarma PS, Thankappan KR. Community-based group intervention for tobacco cessation in rural Tamil Nadu, India: a cluster randomized trial. J Subst Abuse Treat. 2012;43(1):53-60. doi:10.1016/j.jsat.2011.10.026

7. Thankappan KR. Tobacco cessation in India: A priority health intervention. Indian J Med Res. 2014;139(4):484486. PMID:25055394.

8. Raw M, Mackay J, Reddy S. Time to take tobacco dependence treatment seriously. Lancet. 2016;387(10017):412-413. doi:10.1016/S0140-6736(15)00950-2

9. Thankappan KR, Pradeepkumar AS, Nichter M. Doctors' behaviour \& skills for tobacco cessation in Kerala. Indian J Med Res. 2009;129(3):249-255. PMID:19491416.

10. Whittaker R, McRobbie H, Bullen C, et al. Mobile phone-based interventions for smoking cessation. Cochrane Database Syst Rev. 2016;4:CD006611. doi:10.1002/14651858.CD006611.pub4

11. Noonan D, Silva S, Njuru J, et al. Feasibility of a text-based smoking cessation intervention in rural older adults. Health Educ Res. 2018;33(1):81-88. doi:10.1093/her/cyx080

12. Ministry of Health and Family Welfare, Government of India. mCessation Programme - Quit Tobacco for Life. https://www.nhp.gov.in/quit-tobacco-aboutprogramme_mtl. Published December 23, 2015. Accessed December 31, 2019.

13. Gopinathan P, Kaur J, Joshi S, et al. Self-reported quit rates and quit attempts among subscribers of a mobile text messaging-based tobacco cessation program in India. BMJ Innovations. 2018;4(4):147-154. doi:10.1136/bmjinnov-2018-000285

14. Sealed Envelope Ltd. Power calculator for binary outcome superiority trial. https://www.sealedenvelope.com/ power/binary-superiority/. Published 2012. Accessed March 15, 2020.
15. Sharma R. Revision of Prasad's social classification and provision of an online tool for real-time updating. South Asian J Cancer. 2013;2(3):157. doi:10.4103\%2F2278-330X.114142

16. Parashar M, Singh M, Agarwalla R, Panda M, Pathak R. Predictors of intention to quit tobacco among construction site workers in Delhi, India. Indian J Psychiatry. 2017;59(2):208-213. doi:10.4103/psychiatry.IndianJPsychiatry_368_16

17. Ahluwalia IB, Smith T, Armour BS. Current Tobacco Smoking, Quit Attempts, Knowledge About Smoking Risks Among Persons Aged > 15 Years - Global Adult Tobacco Survey, 28 Countries, 2008-16. MMWR. 2018;67(38):1072-1076. doi:10.15585/mmwr.mm6738a7

18. Yildiz A, Unlu B, Yurdalan U. The attitudes of healthy people about smoking and their knowledge about smoking cessation. Eur Respir J. 2017;50(suppl61):PA2666. doi:10.1183/1393003.congress-2017.pa2666

19. Institute of Medicine (US) Committee on Health and Behavior: Research, Practice, and Policy. Health and Behavior: The Interplay of Biological, Behavioral, and Societal Influences. Washington, DC: National Academies Press (US); 2001. PMID:20669491.

20. Goel S, Verma M, Singh RJ, Bhardwaj AK. Integrating tobacco and tuberculosis control programs in India: A win-win situation. Int J Non-Commun Dis. 2018;3(Suppl S1):9-15. doi:10.4103/jncd.jncd_15_18

CONFLICTS OF INTEREST

The authors have completed and submitted the ICMJE Form for Disclosure of Potential Conflicts of Interest and none was reported.

FUNDING

There was no source of funding for this research.

\section{AUTHORS' CONTRIBUTIONS}

PY, SB and BB contributed to the conception and design of the study. SB performed the statistical analysis and drafted the manuscript. PY and $A Y$ conducted data collection and management. BB supervised the whole study process. All authors read and approved the final manuscript.

PROVENANCE AND PEER REVIEW

Not commissioned; externally peer reviewed. 